\title{
Modelling of particle paths passing through an ultrasonic standing wave
}

\author{
R.J. Townsend ${ }^{a}$, M. Hill ${ }^{\mathrm{a}, *}$, N.R. Harris ${ }^{\mathrm{b}}$, N.M. White ${ }^{\mathrm{b}}$ \\ a School of Engineering Sciences, Electromechanical Research Group, University of Southampton, Southampton SO17 1BJ, UK \\ ${ }^{\mathrm{b}}$ Electronics and Computer Science, University of Southampton, Southampton SO17 1BJ, UK
}

\begin{abstract}
Within an ultrasonic standing wave particles experience acoustic radiation forces causing agglomeration at the nodal planes of the wave. The technique can be used to agglomerate, suspend, or manipulate particles within a flow. To control agglomeration rate it is important to balance forces on the particles and, in the case where a fluid/particle mix flows across the applied acoustic field, it is also necessary to optimise fluid flow rate.

To investigate the acoustic and fluid forces in such a system a particle model has been developed, extending an earlier model used to characterise the 1-dimensional field in a layered resonator. In order to simulate fluid drag forces, CFD software has been used to determine the velocity profile of the fluid/particle mix passing through the acoustic device. The profile is then incorporated into a MATLAB model. Based on particle force components, a numerical approach has been used to determine particle paths. Using particle coordinates, both particle concentration across the fluid channel and concentration through multiple outlets are calculated.

Such an approach has been used to analyse the operation of a microfluidic flow-through separator, which uses a half wavelength standing wave across the main channel of the device. This causes particles to converge near the axial plane of the channel, delivering high and low particle concentrated flow through two outlets, respectively. By extending the model to analyse particle separation over a frequency range, it is possible to identify the resonant frequencies of the device and associated separation performance.

This approach will also be used to improve the geometric design of the microengineered fluid channels, where the particle model can determine the limiting fluid flow rate for separation to occur, the value of which is then applied to a CFD model of the device geometry.
\end{abstract}

(C) 2004 Elsevier B.V. All rights reserved.

Keywords: Radiation force; CFD; Standing waves; Concentration

\section{Introduction}

Technologies are being studied which exploit acoustic radiation forces on particles within standing waves. Such technologies include particle separation, fractionation and agglomeration, finding applications in areas such as biotechnology [1,2], material processing $[3,4]$ and filter systems.

For particle manipulation techniques, it is important to understand how particle motion is influenced by factors including the acoustic field and fluid motion. By considering the various forces experienced by particles \footnotetext{
3053.

${ }^{*}$ Corresponding author. Tel.: +44-23-8059-3075; fax: +44-23-8059-

E-mail address: m.hill@soton.ac.uk (M. Hill).
}

and being able to balance them, it is possible to control their movement and agglomeration rate.

Particle motion can be predicted by considering the principal fluid and acoustic forces, manipulated and solved numerically [5,6] or coded into CFD models [7]. This paper describes a similar approach where particle movement is predicted by summating particle forces and solving the resulting equations numerically. However, the method described aims to include more accurate descriptions of the fluid velocity profile and acoustic radiation force, accounting for the layered nature of ultrasound devices. This approach is generally applicable for ultrasonic devices which exploit acoustic radiation forces, although here an emphasis has been placed on those involving fluid flow through. An ultrasonic separator is used as a specific example, demonstrating the application of the model. 


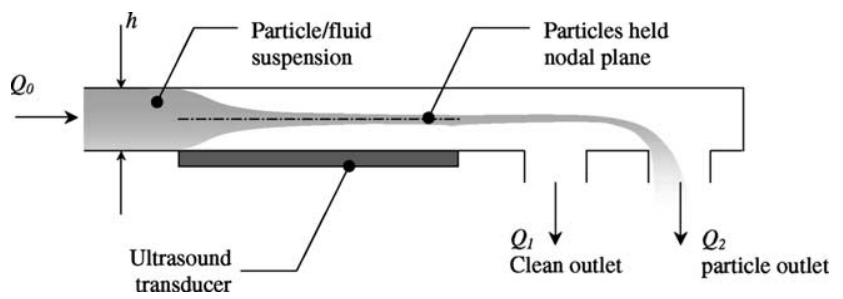

Fig. 1. Schematic of separation device operating in half wavelength mode.

\section{Prediction of particle motion and concentration}

\subsection{Cell parameters}

A microfluidic ultrasonic separator is used as an example application in this paper (Fig. 1). At certain frequencies resonance will be induced in the fluid layer. When the transducer is driven at $\sim 3 \mathrm{MHz}$, a half wavelength mode is excited and a standing wave is produced across the fluid channel, i.e. the height of the fluid microchannel is approximately half the acoustic wavelength, $h=\lambda / 2$ As particles of radius $R$ pass through the standing wave they are forced onto the nodal plane and extracted through the second of two outlets with flow rates $Q_{1}$ and $Q_{2}$, respectively, and where $Q_{2}>Q_{1}$.

In the following text, typical values are applied and include $h=250 \mu \mathrm{m}$, yeast particle radius $R=3 \mu \mathrm{m}$ and total flow rate $Q_{0}=0.1 \mathrm{ml} / \mathrm{s}$. Water is used as the working fluid where density $\rho=1000 \mathrm{~kg} / \mathrm{m}^{3}$ and dynamic viscosity $\mu=0.001 \mathrm{~kg} / \mathrm{ms}$.

\subsection{Particle forces}

To determine the motion of particles through the microchannel and acoustic field, the system of forces on a particle is considered. The dominant forces are discussed:

\subsubsection{Acoustic radiation force}

Within an acoustic field the time averaged acoustic force on a particle, $F_{\mathrm{ac}}$, in a direction normal to the transducer is given by [8]:

$\left\langle F_{\mathrm{ac}}(x, y, z)\right\rangle=-\nabla\left\langle\phi^{\mathrm{G}}(x, y, z)\right\rangle$,

with

$\left\langle\phi^{\mathrm{G}}(x, y, z)\right\rangle=-V\left[\frac{3\left(\rho_{\mathrm{p}}-\rho_{\mathrm{f}}\right)}{2 \rho_{\mathrm{p}}+\rho_{\mathrm{f}}}\left\langle\bar{E}_{\mathrm{kin}}\right\rangle-\left(1-\frac{c_{\mathrm{f}}^{2} \rho_{\mathrm{f}}}{c_{\mathrm{p}}^{2} \rho_{\mathrm{p}}}\right)\left\langle\bar{E}_{\mathrm{pot}}\right\rangle\right]$,

where $V$ is particle volume, $\rho_{\mathrm{p}}$ and $\rho_{\mathrm{f}}$ are the particle and fluid densities, $c_{\mathrm{p}}$ and $c_{\mathrm{f}}$ represent speed of sound for the particle and fluid mediums, $\left\langle\bar{E}_{\text {kin }}\right\rangle$ and $\left\langle\bar{E}_{\text {pot }}\right\rangle$ are the time-averaged kinetic and potential energies at a point in the field and $\phi^{\mathrm{G}}$ is the gradient of radiation force potential.

Using an acoustic impedance transfer model $[9,10]$, the variation in acoustic velocity and pressure through the device can be determined. The acoustic velocity and pressure are then used to derive the kinetic and potential energies, $\bar{E}_{\text {kin }}$ and $\bar{E}_{\text {pot }}$, respectively, which in turn are used to calculate the variation in $F_{\mathrm{ac}}$ across the fluid layer. As the velocity and pressure fields are dependent on acoustic frequency, the radiation force can also be calculated as a function of frequency, therefore its use is not limited to the resonant case.

\subsubsection{Fluid drag}

For low particle Reynold's number, $R e_{\mathrm{p}}<0.2$, the drag force $F_{\mathrm{D}}$ on a particle is calculated using Stokes drag, where $A$ is the area of the particle in a plane normal to the flow and the velocity $U_{0}$ is the relative velocity between the fluid, $U(x, y)$, and particle, $u(x, y)$ :

$F_{\mathrm{D}}=\frac{1}{2} C_{\mathrm{D}} \rho_{\mathrm{f}} U_{0}^{2} A$,

where

$C_{\mathrm{D}}=\frac{24}{R e} \quad$ and $\quad R e_{\mathrm{p}}=\frac{2 R \mu U_{0}}{\rho_{\mathrm{f}}}$.

Combining $u(x, y)$ and $U(x, y)$ with Eq. (3) and expressions for $C_{\mathrm{D}}$ and $R e_{\mathrm{p}}$ gives:

$F_{\mathrm{D}}(x, y)=6 \mu \pi R(U(x, y)-u(x, y))$

\subsubsection{Buoyancy}

The relative densities of the particle and fluid control the buoyancy force on a particle, where $F_{\mathrm{B}}$ is opposite to the gravitational force:

$F_{\mathrm{B}}=\frac{4}{3} \pi R^{3} g\left(\rho_{\mathrm{f}}-\rho_{\mathrm{p}}\right)$.

When referenced to the $x$ and $y$ coordinates of the cell, the $x$ and $y$ components of the buoyancy force depend on the orientation of the channel.

\subsubsection{Other particle forces}

In addition to acoustic radiation and drag forces, particles experience lift [11], van der Waals forces operating in the region of the channel walls [12] and interparticle forces [8]. These forces are generally small compared to the dominant forces already discussed and so, at this stage, have not been included in the modelling reported here.

\subsubsection{Summary of forces}

The acoustic radiation force $F_{\mathrm{ac}}$ operates only in the $y$ direction (ignoring second order effects), whilst drag, $F_{\mathrm{D}}$ and buoyancy $F_{\mathrm{B}}$ can be split into $x$ and $y$ components. They are represented in Fig. 2. 


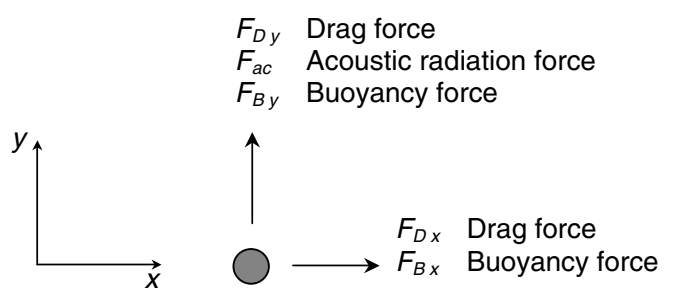

Fig. 2. System of particle forces within fluid suspension and acoustic field.

\subsection{Velocity profile through channel}

\subsubsection{CFD simulations}

Accurate characterisation of the laminar flow velocity profile is fundamental to determining the particle drag force, for which a CFD model is used. In order to validate the CFD model a simple case, based on $h=240 \mu \mathrm{m}$ and flow rate $Q_{0}=0.1 \mathrm{ml} / \mathrm{s}$, has been modelled and compared with classical flow theory.

Due to the low Reynold's number, typically $R e=20$ using $h$ as the characteristic depth $d$, the entry length is small (distance before which steady velocity profile develops) and is of the order $0.29 \mathrm{~mm}$, as calculated below:

$\frac{L_{\mathrm{e}}}{d} \approx 0.06 R e$.

Beyond a distance $L_{\mathrm{e}}$ the flow becomes fully developed, where the fully developed velocity profile $U_{\mathrm{x}}$ between parallel plates is described by a parabolic function [13] and where $\bar{U}$ is the mean fluid velocity:

$U_{\mathrm{x}}=\frac{6 \bar{U}}{h^{2}}\left(h y-y^{2}\right)$.

The channel has been modelled in CFD software where after a distance equal to $L_{\mathrm{e}}$ flow becomes fully developed. Fig. 3 compares the fully developed velocity

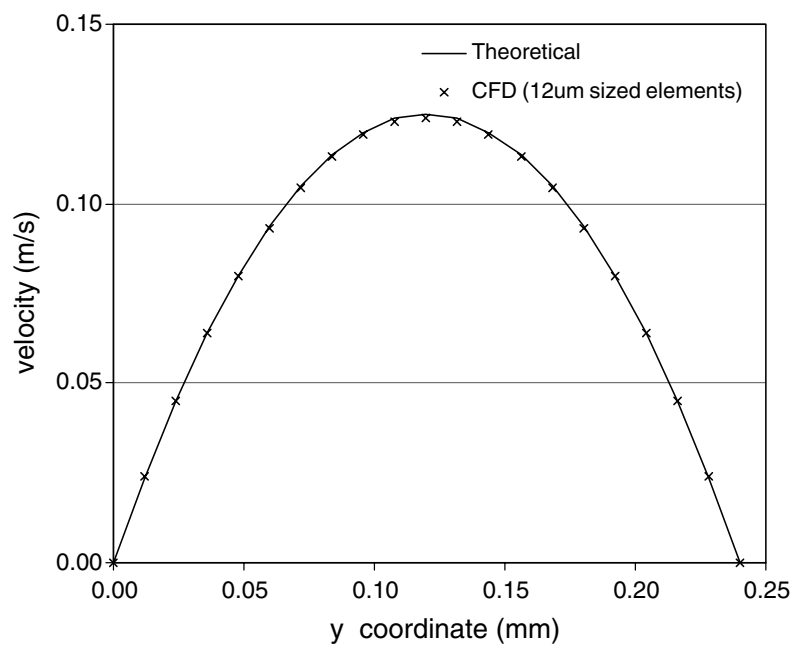

Fig. 3. Theoretical and CFD modelled velocity profile across channel, where $h=240 \mu \mathrm{m}$ and $\bar{U}=0.083 \mathrm{~m} / \mathrm{s}\left(Q_{0}=0.1 \mathrm{ml} / \mathrm{s}\right)$. profile calculated by theory with that determined by CFD using $12 \mu \mathrm{m}$ sized elements.

It can be seen that the CFD derived velocity profile provides a close match with theory, where the element size chosen corresponds to an error $<1 \%$. For instances where more complex flow patterns occur within the channel (eddy regions and entry region flow) and which cannot be characterised by classical theory, CFD simulations can be used to describe the velocity field. Once converted into look-up tables, this velocity field data can be integrated into the numerical software model discussed later.

\subsubsection{Acoustic streaming}

Acoustic streaming occurs when an acoustic wave passes through a fluid medium, where acoustic energy is dissipated into the fluid in the form of momentum and causes steady fluid flow. Of the types of streaming applicable to microchannels, Rayleigh streaming occurs in the fluid bulk producing eddy flows, the size in the order of $\lambda$, and also Schlichting streaming, which influences boundary layer flow [14].

Acoustic streaming patterns will influence the velocity profile through the channel. Although not included in the modelling described, the streaming flow field can simply be superimposed onto the laminar flow profile calculated using CFD.

\subsection{Numerical solver to determine particle trajectory}

To calculate the motion of a particle of mass $m$, the force terms are summed to give accelerations $\ddot{x}$ and $\ddot{y}$ :

$$
\begin{aligned}
& \ddot{x}=\frac{\sum F_{\mathrm{x}}}{m}=\frac{F_{\mathrm{Dx}}+F_{\mathrm{Bx}}}{m} \\
& \ddot{y}=\frac{\sum F_{\mathrm{y}}}{m}=\frac{F_{\mathrm{ac}}+F_{\mathrm{Dy}}+F_{\mathrm{By}}}{m}
\end{aligned}
$$

The option of developing an analytical solution to describe the motion of a particle through a standing wave exists by solving only Eq. (4) and a special case of (1) describing an 'ideal' standing wave, shown below:

$$
F_{\mathrm{ac}}=4 \pi\langle\bar{\varepsilon}\rangle k R^{3}\left[\frac{\rho_{\mathrm{p}}+\frac{2}{3}\left(\rho_{\mathrm{p}}-\rho_{\mathrm{f}}\right)}{2 \rho_{\mathrm{p}}+\rho_{\mathrm{f}}}-\frac{1}{3} \frac{c_{\mathrm{f}}^{2} \rho_{\mathrm{f}}}{c_{\mathrm{p}}^{2} \rho_{\mathrm{p}}}\right] \sin (2 k y) \text {. }
$$

Additionally, for a near neutrally buoyant particle (e.g. bacteria in water) the buoyancy force tends to zero.

However, when considering resonance within a layered device where the 'ideal' standing wave case is not necessarily applicable, the acoustic force described by Eq. (1) should be used and has been implemented using a numerical approach.

An acoustic description of such a separator device has been modelled numerically by Hill et al. [10] in 
MATLAB and uses Eq. (1) to calculate the radiation force. This model has since been developed to include other particle forces and summates these forces as expressed by Eqs. (8) and (9). These equations are then solved using an ODE numerical solver function within MATLAB; this approach ensures that developments to the model, specifically inclusion of other particle forces, can be incorporated easily without requiring potentially complex algebraic manipulation. From the ODE solver, the model provides an output array containing a series of time intervals with associated particle coordinate locations and velocity components. The coordinate locations describe the trajectory of a particle, examples of which are shown in Fig. 4.

The particle trajectories are shown in conjunction with the acoustic radiation force and also illustrate the influence of the laminar flow profile by comparing the particle trajectory with 'plug' flow (uniform fluid velocity). The rate of convergence towards the node is not influenced by the longitudinal flow profile, assuming the effects of lift to be negligible. However, for the parabolic profile the low flow rate at the wall causes the particles to begin converging at a position further upstream as compared to plug flow. This demonstrates the significant influence the flow profile has on the particle trajectory and therefore the implications on design and resulting efficiency of the device.

It can be seen that the acoustic radiation force is not zero at the $y=0$ channel wall, as would be predicted by Eq. (10), as the method used in the model considers the influence of the layered nature of the device [10]. Further, the nodal plane will not necessarily be located along the centre plane of the channel, but its position can be predicted.

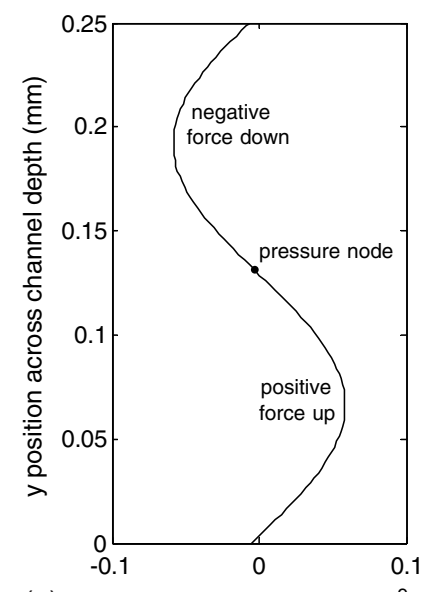

(a) Acoustic radiation force $\left(\times 10^{9} \mathrm{~N}\right)$

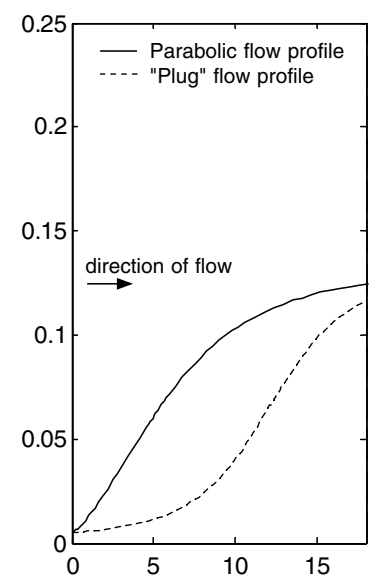

(b) $x$ position along length of channel $(\mathrm{mm})$

Fig. 4. (a) Acoustic radiation force and (b) trajectory of a particle initially located near channel wall for both parabolic flow and 'plug' flow profiles, where $h=250 \mu \mathrm{m}$.

\subsection{Particle concentration}

By modelling a series of particles at the channel inlet and using the resulting particle coordinates, it is possible to calculate the change in concentration across the channel. The change in concentration is dependent on the change in lateral $(x)$ spacing between each particle. Additionally, the velocity profile influences the concentration; as a stream of particles moves into planes of increased fluid velocity, the longitudinal $(y)$ spacing between particles must increase for particle flow rate to remain constant according to continuity.

Fig. 5 illustrates the convergence of a series of particles towards the nodal plane. At a distance $8.5 \mathrm{~mm}$ along the acoustic field the relative concentration of particles across the channel is calculated and plotted.

The asymmetric nature of the acoustic radiation force across the channel is apparent as a small number of particles are forced to the lower wall. This identifies a pressure anti-node which exists within the fluid channel and from which particles are repelled. However, the majority of particles are forced towards the centre of the channel where a peak in particle concentration can be seen.

The concentration through an outlet is calculated by determining particle flow rate between the wall and position at which the flow splits between outlets. Dividing this by the corresponding outlet fluid flow rate results in particle concentration.

\subsection{Frequency dependency}

As previously discussed, the acoustic radiation force can be modelled as a function of frequency, therefore
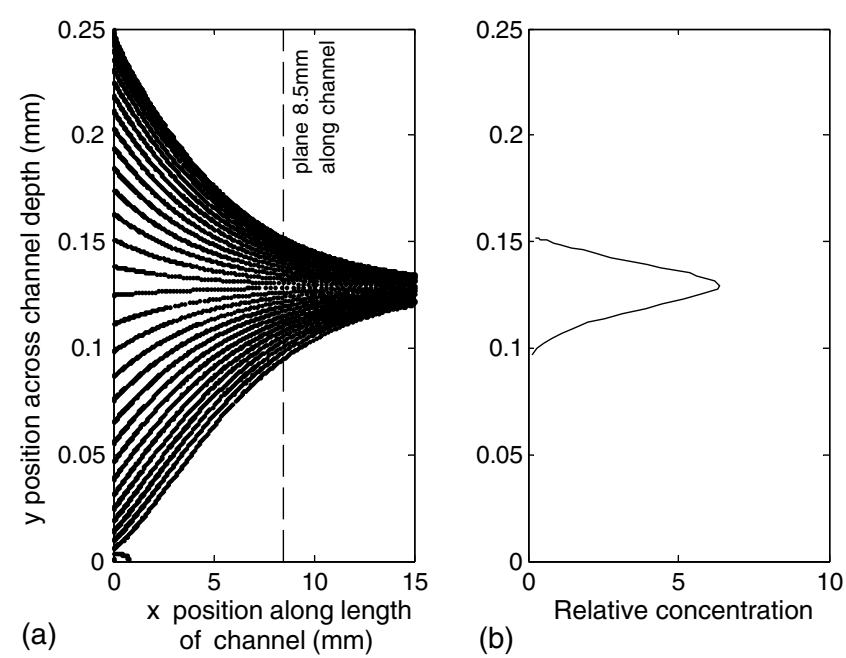

(b)

Fig. 5. (a) Convergence of particles towards nodal plane and (b) relative particle concentration across channel at a distance $8.5 \mathrm{~mm}$ along channel and acoustic field (relative concentration at inlet $=1$ ) and where $h=250 \mu \mathrm{m}$. 


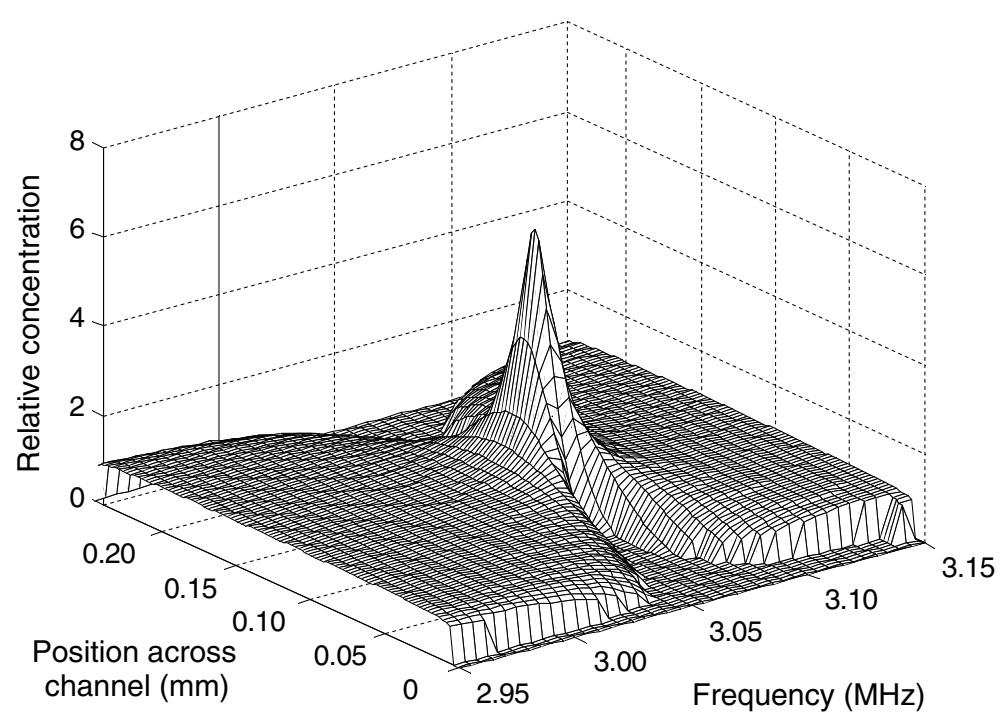

Fig. 6. Concentration across channel as function of frequency taken at a distance $8.5 \mathrm{~mm}$ along channel and where $h=250 \mu \mathrm{m}$.

particle concentration can be plotted against frequency in addition to channel position, $y$. Fig. 6 shows an example of how the concentration can vary as function of frequency.

The peak seen at a frequency $\sim 3.06 \mathrm{MHz}$ corresponds to a resonant frequency of the device where the radiation force is a maximum. Moving away from this frequency, the radiation force decreases until the particle concentration ceases to change relative to the inlet concentration. Similarly, the relationship between outlet concentration and frequency can be plotted.

\subsubsection{Separation test results}

A similar device has been tested using the experimental apparatus described by Hawkes and Coakley [6] and concentration through outlet 1 is measured. Fig. 7 shows the concentration measured between frequencies 2.8 and $3 \mathrm{MHz}$. This is compared to the modelled outlet concentration.

Some discrepancies can be seen where during experiment particles often form into clumps caused by second

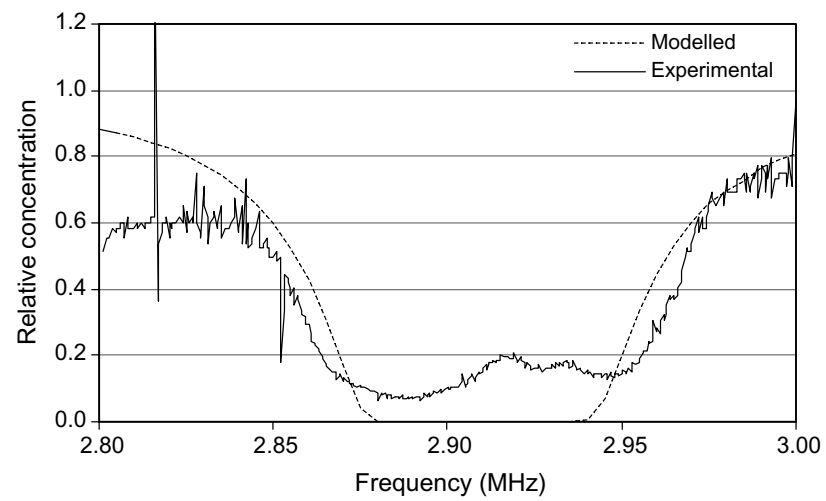

Fig. 7. Concentration through outlet 1 (clean fluid) as a function of frequency, comparing experimental results and modelled performance. order acoustic forces [8]. This will influence the measured concentration and is likely to give an inaccurate representation of the true concentration, making the signal appear noisy at higher concentration levels. Any inaccuracy in the measurement of channel depth, $h$, influences the resonant frequency predicted by the model and will shift the plot along the $x$-axis. However, the model offers a reasonable prediction of the particle concentration and behavior of particles within the device.

\section{Application of model}

Generally, the model can be used to predict the influence of various parameters on the agglomeration rate of particles and therefore the influence on separation efficiency or agglomerate concentration, depending on the application of the device. For flow-through applications the model can be used to optimise flow rates and acoustic energy density to maximise the device efficiency, therefore minimising power requirements by the transducer and, for example, avoiding deleterious heating effects.

\section{Conclusion}

The ability to predict particle concentration levels within an ultrasonic device has been demonstrated, based on a numerical approach to determine particle trajectories. The method has been tested predicting the performance of an ultrasonic separator and can also be used to model macrofluidic as well as microfluidic devices, assuming laminar flow to be applicable. The model can also be adapted to tackle any number of outlets. 
It is known that other particle forces and second order acoustic effects, not yet included, influence the motion of particles, but generally these can be incorporated into the model with minimum simplification and manipulation.

\section{Acknowledgements}

This work is funded by EPSRC grant GR/R13333/01. The authors gratefully acknowledge the funding and support from Dstl and Porvair Plc. and also Dr. Hawke's assistance with experimental work.

\section{References}

[1] W.T. Coakley, J.J. Hawkes, M.A. Sobanski, C.M. Cousins, J. Spengler, Analytical scale ultrasonic standing wave manipulation of cells and microparticles, Ultrasonics 38 (2000) 638-641.

[2] R. Karumanchi, S.N. Doddamane, C. Sampangi, P.W. Todd, Field-assisted extraction of cells, particles and macromolecules, Trends in Biotechnology 20 (2002) 72-78.

[3] Y. Abe, M. Kawaji, T. Watanabe, Study on the bubble motion control by ultrasonic wave, Experimental Thermal and Fluid Science 26 (2002) 817-826.
[4] S. Gupta, D.L. Feke, I. Manaszloczower, Fractionation of mixed particulate solids according to compressibility using ultrasonic standing-wave fields, Chemical Engineering Science 50 (1995) 3275-3284.

[5] D.A. Johnson, D.L. Feke, Methodology for fractionating suspended particles using ultrasonic standing wave and divided flow fields, Separations Technology 5 (1995) 251-258.

[6] J.J. Hawkes, W.T. Coakley, Force field particle filter, combining ultrasound standing waves and laminar flow, Sensors and Actuators B-Chemical 75 (2001) 213-222.

[7] I.L.J. Holwill, The use of ultrasonic standing waves to enhance optical particle sizing equipment, Ultrasonics 38 (2000) 650-653.

[8] M. Gröschl, Ultrasonic separation of suspended particles-Part I: Fundamentals, Acustica 84 (1998) 432-447.

[9] M. Hill, R.J.K. Wood, Modelling in the design of a flow-through ultrasonic separator, Ultrasonics 38 (2000) 662-665.

[10] M. Hill, Y.J. Shen, J.J. Hawkes, Modelling of layered resonators for ultrasonic separation, Ultrasonics 40 (2002) 385-392.

[11] M.T. Lawler, P. Lu, The role of lift in the radial migration of particles in a pipe flow, in: I. Zandi (Ed.), Advances in solid-liquid flow in pipes and its applications, Pergamon Press, New York, 1971, pp. 39-57.

[12] Q. Qi, G.J. Brereton, Mechanisms of removal of micron-sized particles by high-frequency ultrasonic-waves, IEEE Transactions on Ultrasonics Ferroelectrics and Frequency Control 42 (1995) 619-629.

[13] F.M. White, Fluid Mechanics, 4th ed., McGraw-Hill, 1999.

[14] L.K. Zarembo, Acoustic streaming, in: L.D. Rozenburg (Ed.), High Intensity Ultrasonic Fields, Plenum Press, New York, 1971, pp. 137-199. 